\title{
Overcoming horizontal depolarizing resonances with multiple tune jumps
}

\author{
H. Huang, L. A. Ahrens, M. Bai, K. A. Brown, Y. Dutheil, C. Gardner, J. W. Glenn, F. Lin, \\ W. W. MacKay, F. Meot, A. Poblaguev, V. Ranjbar, T. Roser, V. Schoefer, S. Tepikian, \\ N. Tsoupas, K. Yip, A. Zelenski, and K. Zeno \\ Brookhaven National Laboratory, Upton, New York 11973, USA
}

(Received 2 May 2014; published 11 August 2014)

\begin{abstract}
In a medium energy proton synchrotron, strong enough partial Siberian snakes can be used to avoid both imperfection and vertical intrinsic depolarizing resonances. However, partial snakes tilt the stable spin direction away from vertical, which generates depolarizing resonances associated with horizontal tune. The relatively weak but numerous horizontal intrinsic resonances are the main source of the residual polarization losses. A pair of horizontal tune jump quads have been used in the Brookhaven Alternating Gradient Synchrotron to overcome these weak resonances. The locations of the two quads have to be chosen such that the disturbance to the beam optics is minimum. The emittance growth has to be mitigated for this method to work. In addition, this technique needs very accurate jump timing. Using two partial Siberian snakes, with vertical tune inside the spin tune gap and $80 \%$ polarization at the Alternating Gradient Synchrotron injection, polarized proton beam had reached $1.5 \times 10^{11}$ proton per bunch with $65 \%$ polarization. With the tune jump timing optimized and emittance preserved, more than $70 \%$ polarization with $2 \times 10^{11}$ protons per bunch has been achieved. The polarization transport efficiency is close to $90 \%$.
\end{abstract}

DOI: 10.1103/PhysRevSTAB.17.081001

PACS numbers: 41.75.Ak, 29.27.Bd, 29.27.Hj

\section{INTRODUCTION}

Polarized proton beams encounter numerous depolarizing resonances during acceleration in synchrotron accelerators. Polarization preservation requires careful manipulation of both orbital motion and spin motion. During acceleration, two types of depolarizing resonances are crossed whenever the spin precession frequency equals the frequency with which spin-perturbing magnetic fields are encountered. The spin precession frequency is defined as the number of full spin precessions per orbital revolution and is also called spin tune $\nu_{s p}$. For a synchrotron with only vertical bending field, spin tune is equal to $G \gamma$, where $G$ is anomalous magnetic moment of the beam particles, $\gamma$ is Lorentz factor. For proton, $G=1.7928$. The resonance condition for imperfection depolarizing resonances arises when $\nu_{s p}=n$, where $n$ is an integer. The condition for intrinsic resonances is $\nu_{s p}=k P \pm \nu_{y}$ where $k$ is an integer, $\nu_{y}$ is the vertical betatron tune, and $P$ is the superperiodicity. Traditionally, the intrinsic resonances are overcome by using a vertical betatron tune jump, which effectively makes the resonance-crossing rate large, and the imperfection resonances are overcome with the harmonic corrections of the vertical orbit to reduce the resonance strength. This has been done at ZGS, KEK, AGS, and

"Present address: Jefferson Lab, Newport News, VA 23606.

Published by the American Physical Society under the terms of the Creative Commons Attribution 3.0 License. Further distribution of this work must maintain attribution to the author $(s)$ and the published article's title, journal citation, and DOI.
COSY [1-3]. As the beam energy increases, these traditional methods become difficult and time consuming.

Over the past twenty years, Siberian snake [4] magnets have been invoked to overcome the depolarizing resonances [5-6]. Since full snake generates intolerable orbit distortion in the medium energy synchrotron, partial snake [7-8] is the choice for medium energy range (5-25 GeV). A dual partial snake scheme [9] has been used in the AGS to overcome both imperfection and vertical intrinsic resonances. The two partial snakes are separated by $1 / 3$ of ring circumference. In this case, the total snake strength is modulated with a multiple of 3 and maximized when $G \gamma=3 n$, where $n$ is an integer. The spin tune $\nu_{s p}$ is given by:

$$
\begin{aligned}
\cos \pi \nu_{s p}= & \cos \frac{s_{1} \pi}{2} \cos \frac{s_{2} \pi}{2} \cos G \gamma \pi \\
& -\sin \frac{s_{1} \pi}{2} \sin \frac{s_{2} \pi}{2} \cos \frac{G \gamma \pi}{3},
\end{aligned}
$$

where $s_{1} \pi$ and $s_{2} \pi$ are the rotation angles of the two partial snakes. The snake strength and spin tune modulations perfectly meet the requirement: larger spin tune gap at strong intrinsic resonance locations when a stronger snake strength is needed. When $G \gamma$ is integer, spin tune cannot be an integer hence a spin tune gap is generated. With the spin tune gap, both intrinsic and imperfection resonance can be avoided with proper choice of vertical tune.

For normal synchrotrons, the intrinsic resonance is only associated with the vertical betatron tune $\nu_{y}$ for vertical polarization, as the vertical spin can only be affected by the horizontal magnetic field. However, in the presence of a 
partial snake, the stable spin direction is not purely vertical. Therefore the perturbing fields that rotate the spin away from the stable spin direction have vertical as well as horizontal components. Particles undergoing horizontal betatron oscillations encounter vertical field deviations at the horizontal oscillation frequency. As a result, resonances with the spin tune are driven by the horizontal betatron oscillations, and will occur whenever the spin tune satisfies $G \gamma=k \pm \nu_{x}$, where $k$ is an integer and $\nu_{x}$ is horizontal betatron tune [10]. Since these resonances are relatively weak, a modest tune jump system can suppress them. This paper discusses the experimental results of using modest tune jump system to overcome horizontal intrinsic resonances. The paper is organized as follows. Section II discusses the properties of horizontal intrinsic resonances. Section III discusses the tune jump system and possible emittance growth issue. Section IV gives the operation and beam setup details. Section V discusses the tune jump experimental results and analysis.

\section{HORIZONTAL INTRINSIC RESONANCES}

To avoid these horizontal intrinsic resonances, the horizontal betatron tune can also be put into the spin tune gap generated by the partial snakes. A four partial snake arrangement in AGS which allows the placement of both the horizontal and vertical tunes inside the spin tune gap has been proposed [11], but it requires four equal strength partial snakes. With the existing two partial snakes, there are three problems to keep both tunes inside spin tune gap in the AGS. First of all, it requires stronger partial snakes for a wider spin tune gap, so that the two tunes can stay inside with reasonable tune separation to avoid strong coupling. One snake in the AGS has run at the maximum strength. Increasing the strength of the second one will increase the imbalance between the two and make the spin direction matching at injection and extraction worse. Second, a stronger snake causes larger lattice distortion near injection, which limits the beam intensity. Last but not least, such an optics with both tunes near integer cannot be achieved with existing quadrupole power supplies and an upgrade is required for the horizontal tune quadrupole power supplies. Since all these resonances are relatively weak compared to the vertical counterpart, a modest tune jump system can be built to increase the effective resonance crossing rate to mitigate the polarization loss.

Because the beam is going to be used for Relativistic Heavy Ion Collider (RHIC), the emittance blowup due to the nonadiabatic tune jump has to be small. In addition, since the total polarization loss for the 82 horizontal intrinsic resonances is predicted to be only $15-20 \%$ for the given emittance, it is not possible to determine the jump times by time-scanning each resonance individually while measuring the final polarization, as done in the fast tune jump for strong vertical intrinsic resonances [2]. The timing has to be based on accurate beam energy measurement.

\section{HORIZONTAL TUNE JUMP SCHEME}

These horizontal intrinsic resonances can be divided into two categories: one with $N+\nu_{x}$; one with $N-\nu_{x}$. To increase the crossing speed, the horizontal tune is jumped up (so that $N-\nu_{x}$ going down) when crossing the $N-\nu_{x}$ resonance. It is then jumped down next when crossing $N+\nu_{x}$. Note that the associated vertical tune jump is in the opposite direction of horizontal tune jump. Since $\beta_{y}$ is about half of $\beta_{x}$ at the jump quad location, the vertical tune shift will be half the amplitude of horizontal one. When $G \gamma$ is near integer where imperfection and vertical intrinsic resonances are located, the vertical tune can be back to its nominal value, so that the crossings of these resonances are not affected. The scheme is illustrated in Fig. 1. With partial snakes inserted, spin tune $\nu_{s p}$ is almost linearly proportional to $G \gamma$ except when $G \gamma$ near integer, where a spin tune gap is generated. There are two horizontal intrinsic and two vertical intrinsic resonances for each $G \gamma$ integer interval. The two vertical resonances are avoided by maintaining the vertical tune inside the spin tune gap. The two horizontal intrinsic resonances are overcome by tune jump.

The final polarization after crossing an isolated depolarizing resonance is given by Froissart-Stora formula [12]. For a Gaussian beam, it is given as

$$
\frac{P_{f}}{P_{i}}=\frac{1-\frac{\pi|\epsilon|^{2}}{\alpha}}{1+\frac{\pi|\epsilon|^{2}}{\alpha}}
$$

where $P_{f}$ and $P_{i}$ are the polarization before and after crossing the resonance, respectively, $\epsilon$ is the resonance strength and

$$
\alpha=\frac{d(G \gamma)}{d \theta} \pm \frac{d \nu_{x}}{d \theta}
$$

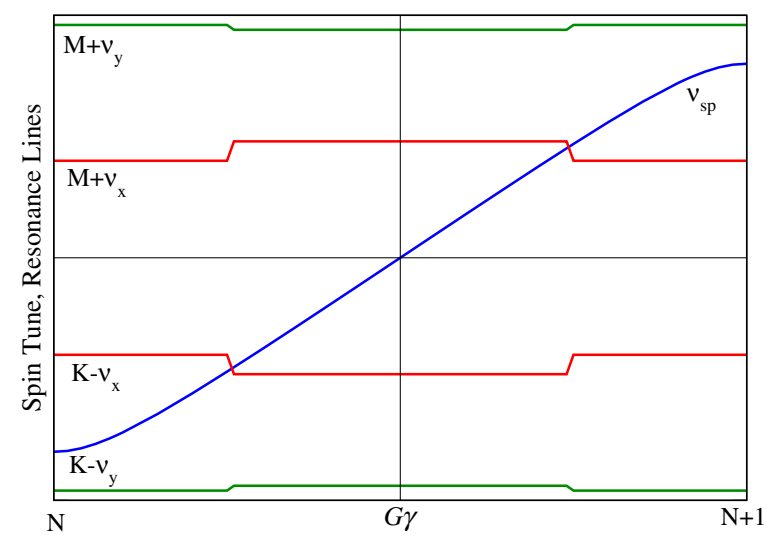

FIG. 1. The schematics of tune jump scheme. The blue trace is the spin tune as function of $G \gamma$ during acceleration. The red lines are the horizontal intrinsic resonance lines. The green lines are vertical resonance lines. Over one unit of $G \gamma$, two horizontal intrinsic resonances are crossed. $\mathrm{M}, \mathrm{K}$, and $\mathrm{N}$ are integers. 
is the resonance crossing rate. For the whole ramp, the final polarization is just the product of the Eq. (2) for all 82 isolated resonances. During normal acceleration in the AGS, the rate of change of $G \gamma$ is about $117 / \mathrm{s}$. The overall polarization loss due to horizontal intrinsic resonances for typical AGS emittance is about $15-20 \%$ over the whole ramp. An increase of the crossing rate by 4 folds will reduce this loss to $4-5 \%$. The gain from further increase is small. To quadruple the resonance crossing speed, a change of $\nu_{x}$ at rate above $0.35 / \mathrm{ms}$ meets the requirement. The AGS horizontal tune jump system can achieve this with a tune jump of 0.04 in $100 \mu \mathrm{s}$.

Compared to vertical tune jump done in the past for polarization purpose [1-3], there are a few differences. First, this tune jump system has to jump many times (up to 82 times for the full AGS ramp), and the effect on polarization from each one is too small to be measurable. This means that the timing has to be dead reckoning. In the best scenario, we can only measure polarization as a function of the overall timing for all resonances. Second, the beam is used for collider injection instead of slow extracted beam. The emittance preservation is important for both polarization preservation and luminosity in the collider. If there is large emittance growth, any polarization gain from the tune jump can be washed out.

The associated 0.02 vertical tune motion occurring over $100 \mu \mathrm{s}$ (about 34 to 37 turns) does pose a problem. Unlike the horizontal tune, which is around 8.70 throughout the ramp, the vertical tune is necessarily close to integer (such as 8.98 or 8.99 ). The vertical betatron motion at the quad is slow relative to the changing of jump quad fields, which makes the field change a nonadiabatic one. Any offset between equilibrium orbit and the jump quad centers generates a kicking which results in emittance growth.

In general, when the number of turns $N$ and tune jump amplitude $\Delta \nu$ satisfy $N \Delta \nu \gg 1$, the process can be viewed as adiabatic. The parameters of the proposed tune jump system are at the border line and simulations are needed. Simulations of emittance evolution with various jump amplitudes and jump quad locations have been done to make sure the emittance growth is small enough due to the tune jump. Particles with a 6-D Gaussian distribution were simulated through the acceleration between $G \gamma=43.5$ and 45.5 where $\nu_{y}$ is the highest (8.99) and the nonadiabaticity is the worst. Several cases are considered in the simulation. The existing beta function beat due to errors is estimated to be about 20\% [13]. This is represented by a single quadrupole in the ring. As the typical horizontal tune is around 8.7 but the vertical tune is close to 8.98 or higher on the ramp, the perturbation to the lattice is expected to be much stronger in the vertical plane. The simulation was done for a single quadrupole and a pair of quadrupoles in straight sections separated by one of 12 AGS superperiods, resulting in a vertical phase advance between the two of nearly $270^{\circ}$. The $270^{\circ}$ separation is necessary because the duration of the tune jump is nonadiabatic with respect to the vertical betatron period: the period is about 100 turns at $\nu_{y}=8.99$. Simulations show that the two quadrupole solution preserves emittance much better than the one quadrupole solution. Simulation also shows that the locations of the quadrupoles are not sensitive to which superperiods are used, as long as the two are separated by one superperiod. A three quadrupole solution was also simulated, but was found to be no better than the two quadrupole solution. Simulations have been done for different number of turns $(1,25,50$ turns) and lattice conditions. From these simulations, we found: (i) The vertical tune jump $\Delta \nu_{y}$ generated by each of the two jump quads has to be equal for the vertical emittance preservation. (ii) There is significant emittance growth for an one turn jump (very nonadiabatic), but there is not much difference between 25 turns and 50 turns. (iii) With $20 \%$ beta function beat included, the vertical emittance growth is about two times larger than without the beta function beat. But it is still below $0.1 \%$. For a total of 82 tune jumps, this translates to no more than $8 \%$ emittance growth. Since the $20 \%$ or less beta function beat is achievable with correction and the vertical tune is not at 8.99 for most part of the ramp, the real emittance growth should be even smaller.

\section{AGS OPERATION AND BEAM SETUP}

The polarized $\mathrm{H}^{-}$beam from the optically pumped polarized ion source was accelerated through $200 \mathrm{MeV}$ linac. The beam polarization at $200 \mathrm{MeV}$ was measured with elastic scattering from a carbon fiber target [14]. The beam polarization was stable and was $80-82 \%$. The beam was then strip-injected and accelerated in the AGS Booster up to $2.36 \mathrm{GeV}$ or $G \gamma=4.5$. At booster injection, the vertical tune is as high as 4.92 to reduce the horizontal beta function at the stripping foil so that emittance growth due to the multiple-turn injection can be minimized. On the booster ramp, the vertical tune was chosen to be 4.8 in order to avoid crossing the intrinsic resonance $G \gamma=\nu_{y}$ in the booster. The imperfection resonances at $G \gamma=3$, and 4 are corrected by using orbit harmonic correction. The beam intensity was scraped in the booster from $5 \times 10^{11}$ down to $2.5 \times 10^{11}$, which reduces beam emittance, mainly in vertical. This is important for beam polarization preservation in the AGS and RHIC. Polarization was measured in the AGS by a Coulomb Nuclear Interference (CNI) polarimeter [15] based on measurement of recoil carbon asymmetry in proton carbon elastic scattering with low momentum transfer (the CNI region).

The AGS injection and extraction energies are chosen as $G \gamma=4.5$ and 45.5 , respectively. At low energies, the helical snake magnets cause significant lattice distortion. Four compensation quads are added around each of the two helical snake magnets, since the quadrupole component of a helical magnet is focusing in both transverse plane. The lattice distortions near injection due to the helical magnets 
are significant. Even with compensation quadrupoles, the vertical tune has to be lower than 8.90 near injection so that the beam size due to beta function distortions does not exceed the aperture limit. The vertical tune is moved into the spin tune gap only after $G \gamma=5$.

\section{A. 6th horizontal harmonics}

The two pulsed quadrupoles that can change the horizontal tune by \pm 0.04 in $100 \mu$ s have been installed in horizontal $\beta_{\max }$ sections, after the fifth combined-function magnet in the I section and J section, named I5 and J5 horizontal tune jump quads. The 12 super periods of AGS are named from A to L. Each super period has 20 combined-function magnets from 1 to 20 . The detail of the tune jump system is given in Ref. [16].

During the beam test, the first observation was that the required currents for the two jump quads differed by a ratio of 3, which implies a big difference in beta functions at the two locations.

The explanation is that a strong $18 \theta$ (two times the tune) vertical beta function beat was created because the tune is close to 9 and the synchrotron superperiod is 12 and not perfect. The effect is well modeled by adding an appropriately (but not uniquely) placed quadrupole into the lattice. The jump quads spacing (1 superperiod, 1/12 of the ring) is $3 / 2$ of an $18 \theta$ wave. If one quad gets a bigger beta, the other gets a smaller beta. Experimentally the effect is large and with following features: (i) it requires the vertical tune to be very high (not seen if $\nu_{y}$ is at 8.9); (ii) it requires significant current in the vertical sextupole string (which is needed for small chromaticity to reduce vertical tune spread and fit in the spin tune gap); (iii) it does not require the snakes.

All these effects can be dramatically reduced by adding some $6 \theta$ horizontal dipole component to the lattice which adds $18 \theta$ quad moment to the lattice by "feed down" in the sextupoles. With this addition, the jump quads gave equal tune shifts for equal currents and orbit response measurements confirm that the beta function beat is substantially reduced. With this change, the jump quads were powered to reach design tune shifts. A survey of the AGS magnets during shutdown was done. A total of nine main magnets have been moved (both radial shift and roll). A few sextupoles have been moved to be aligned with nearby main magnets. The sixth horizontal harmonics have been greatly reduced and the vertical beta beat is consequently smaller. As a validation of the MAD model, the difference of 6th horizontal harmonic amplitude before and after the realignment is calculated and the difference is about the same as the $6 \theta$ orbit harmonic applied during the run to get rid of emittance growth. The measured beta beats before and after correction are shown in Fig. 2. The vertical tune jump amplitude from firing individual quad is about the same afterwards. Figure 3 shows the ratio of vertical tune jump generated by the two jump quads along the

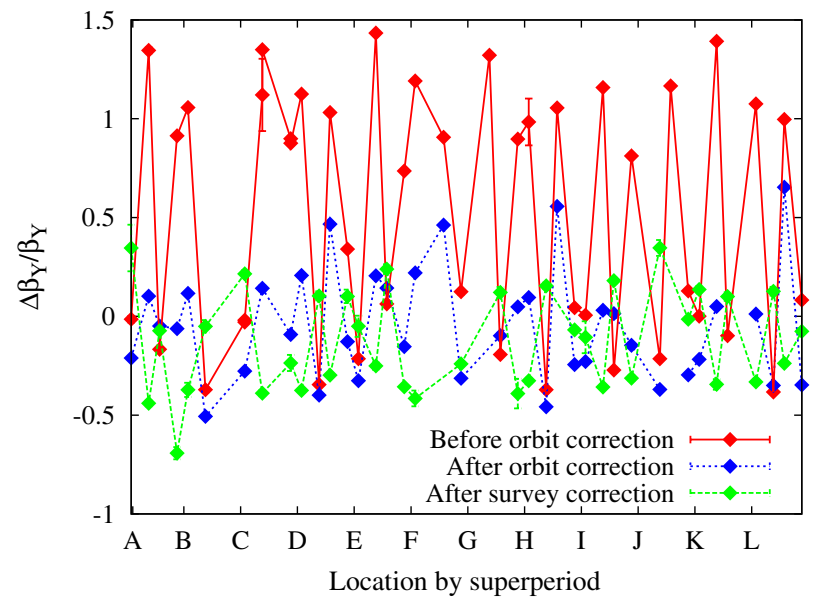

FIG. 2. The measured vertical beta function beat $\left(d \beta_{y} / \beta_{y}\right)$ in the AGS. Zero reading means no beta beat. The beta beat is significantly reduced after the $6 \theta$ horizontal orbit correction or magnet realignment.

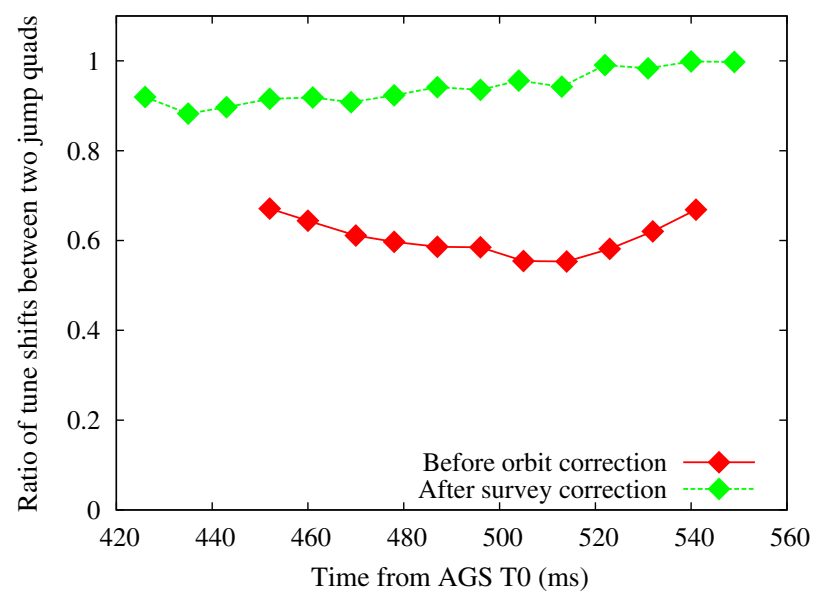

FIG. 3. Ratio of the vertical tune shifts produced by powering each tune jump quad individually at equal current. By design, in a superperiod symmetric AGS, the ratio should be one. AGS acceleration starts at $149 \mathrm{~ms}$ from cycle starting time AGS T0. The acceleration ends at $581 \mathrm{~ms}$ from AGS T0.

AGS ramp as function of time. After the survey, the ratio is close to 1 .

\section{B. Automatic orbit correction and chromaticity}

Each pulsing of the jump quads represents a nonadiabatic change to the closed orbit if the beam is not centered in either of the two tune jump quadrupoles. Since the vertical tune is so close to 9 along the ramp, the vertical orbit is very sensitive to small dipole errors. Over time, the orbit at jump quad locations will drift away from the center of the quads. As the 9th harmonic is the dominant harmonic in the orbit, 9th harmonic correction is the most effective way to correct the orbit. Here the 270 degrees of betatron 


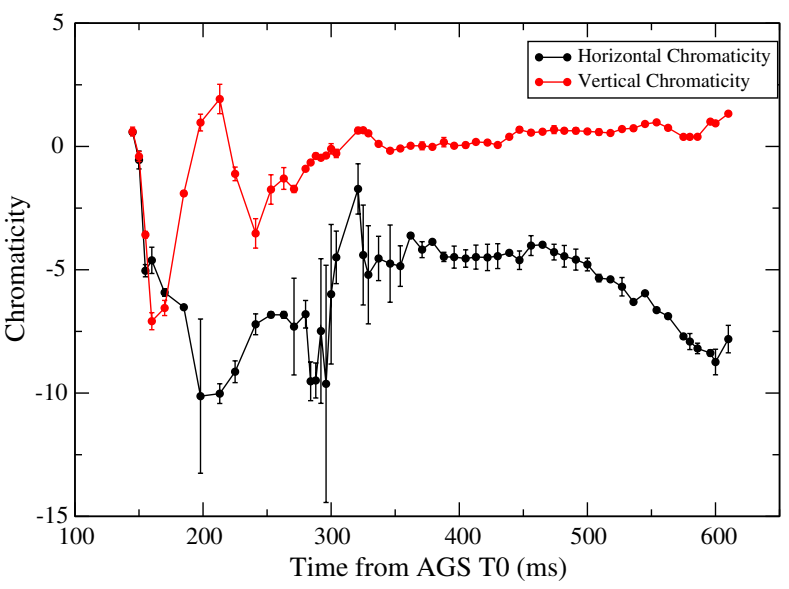

FIG. 4. The measured chromaticities of AGS. The proper values are needed to reduce tune spread and preserve emittance during horizontal tune jump.

phase spacing is valuable as it allows the equilibrium orbit positions at both quads to be adjusted with the two 9th harmonic magnetic phases. Had we spaced the quads by two superperiods this orbit correction scheme would fail. A new feature has been added to the AGS orbit control program which defines the desired 9th harmonics orbit, and "feeds forward" the difference between measured harmonics and the target harmonics so that the difference can be minimized in following AGS cycles.

Even when the vertical tune is put inside the spin tune gap generated by the dual partial snakes, there are still potential polarization losses due to partial snake resonances $[9,17]$. In addition to pushing the vertical tune higher, small tune spread is desired to reduce the footprint of the vertical tune. For the later part of ramp (after $300 \mathrm{~ms}$ ) where resonance strengths are strong, the vertical chromaticity $\left(\xi_{y}=d \nu_{y} /(d p / p)\right)$ is reduced from 2 down to near zero, and the horizontal chromaticity was changed from -10 to about -5 . The measured chromaticities are shown in Fig. 4.

\section{HORIZONTAL TUNE JUMP RESULTS}

With the orbit feed forward, removing the 6th horizontal harmonic and proper chromaticity setup, the emittance is preserved for the horizontal tune jump. Figure 5 illustrates the emittance ratio on the ramp before and after the emittance growth was fixed. The intensity with jump quads on was slightly lower $\left(1.83 \times 10^{11}\right.$ vs $1.87 \times 10^{11}$, just a $2 \%$ difference), but showed no emittance growth.

The measured bunch length and synchrotron frequency give a constant longitudinal emittance of $0.76 \mathrm{eVs}$. This corresponds to $d p / p$ full-width at half-maximum (FWHM) as $2 \times 10^{-3}$ at injection and as $1 \times 10^{-3}$ at flattop. The $d G \gamma$ of beam along the ramp is plotted in Fig. 6. Note that $d G \gamma$ is relatively flat around 0.04 above transition $(300 \mathrm{~ms})$, which is important to the tune jump system (discussed later). In the following discussion, we consider beam particles inside

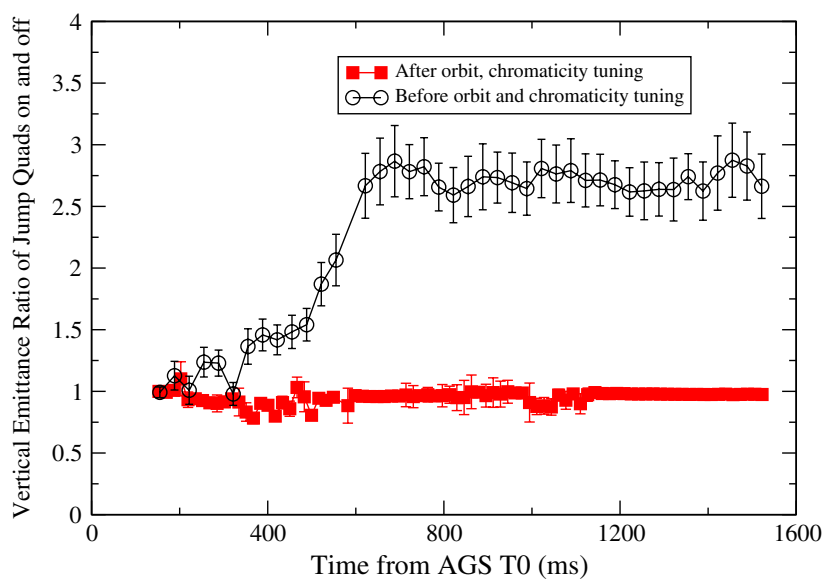

FIG. 5. The measured vertical emittance ratio of jump quads on and off on the AGS ramp. The measurements were done through repeated AGS cycles. The error bars are statistical errors only. The large error bars for the emittance growth case were due to fewer cycles for these measurements.

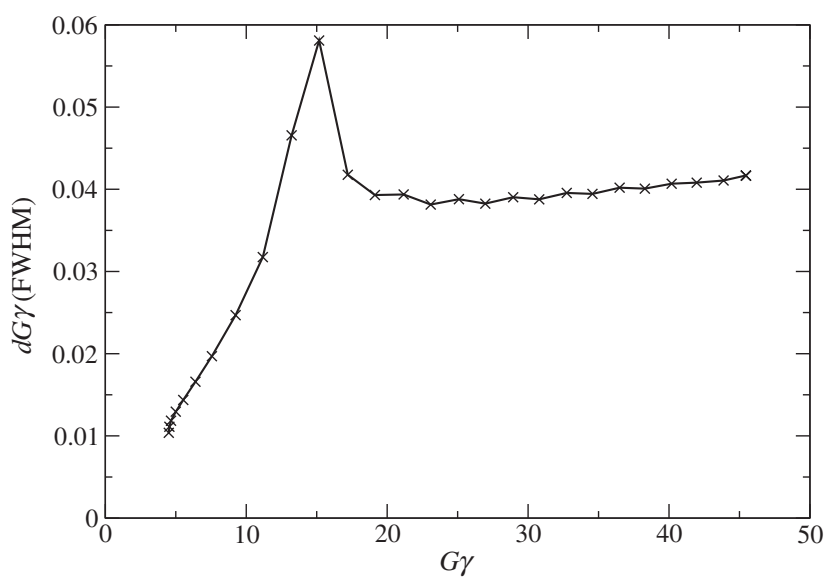

FIG. 6. The $d G \gamma$ FWHM along the ramp as function of $G \gamma$. Note the flatness of the $d G \gamma$ curve beyond $G \gamma=20$. The reduction in $d p / p$ together with the increase in $\mathrm{p}$ result in the spread in the beam $G \gamma$ staying about constant at 0.04 .

the FWHM of the bunch, as about $76 \%$ of beam falls within this width for a Gaussian distribution. In Fig. 1, each resonance was drawn as one line. With nonzero chromaticity and momentum spread, the resonance conditions should be rewritten as

$$
\begin{aligned}
& G \gamma=N-\left(\nu_{x} \pm \xi_{x} d p / p\right), \\
& G \gamma=N+\left(\nu_{x} \pm \xi_{x} d p / p\right),
\end{aligned}
$$

where $\xi_{x}$ is the horizontal chromaticity, $\pm d p / p$ gives the up and down boundaries of the momentum distribution. Ideally, zero chromaticity is needed to eliminate the tune (and resonance condition) dependence on momentum. This is more important for the vertical plane, as vertical intrinsic resonances are stronger. Even though vertical tune is put 


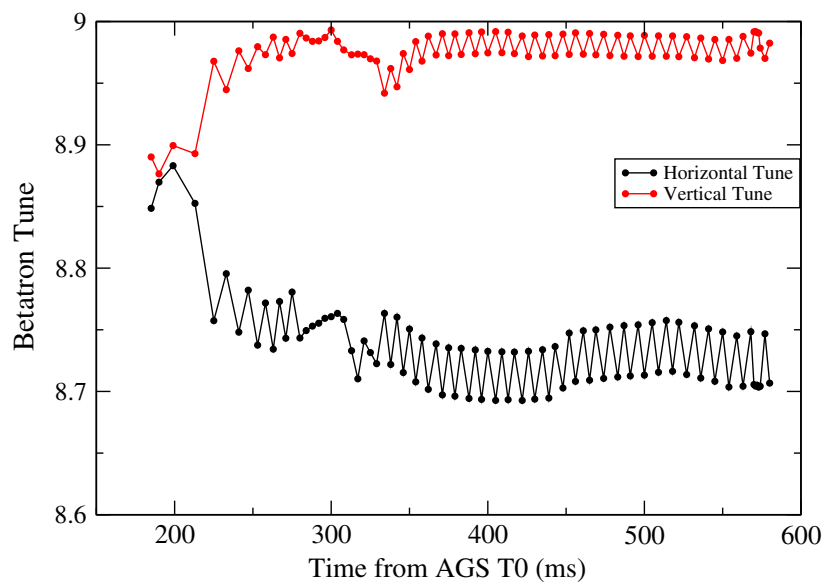

FIG. 7. The measured betatron tunes along the ramp as function of time from AGS T0. The transition is crossed at $313 \mathrm{~ms}$. Note that there was no tune jump around $300 \mathrm{~ms}$ to avoid transition.

into spin tune gap, small tune spread is still required as the higher order partial snake resonances limit the available tune space.

In the AGS, the vertical chromaticity is held near zero to reduce vertical tune spread. Putting both chromaticity near zero results in unstable beam. The horizontal chromaticity is left as negative through the acceleration (as shown in Fig. 4). $\gamma_{t r}$ quads were turned on during the transition crossing to maintain longitudinal beam stability. It was found that firing horizontal tune jump quads during transition crossing interfered with operation of $\gamma_{t r}$ quads, which resulted much stronger longitudinal oscillation after transition. To preserve longitudinal emittance, six pulses(12 jumps) of tune jump pulses were left off around transition. As the result, 70 out of 82 horizontal intrinsic resonances were jumped.

The measured betatron tunes on the AGS ramp as function of time from AGS T0 are shown in Fig. 7. The tune measurement time was chosen such that it gave tunes alternatively as jump up value and jump down value (or no jump value). The ramp starts at $149 \mathrm{~ms}$ and reaches flattop at $581 \mathrm{~ms}$. The figure shows that the horizontal tune jump amplitude is about 0.04 and the vertical tune jump amplitude is about 0.02 .

Figures 8-9 illustrate the resonance crossing with tune and energy spreads. They are plotted for resonances near $G \gamma=45$, but are representative for all resonances when $G \gamma>19$, as $d G \gamma$ remains constant as shown in Fig. 6. To benefit from the tune jump, the beam particles have to cross the resonance line during the jump. For the given beam parameters (tune jump amplitude, chromaticity, beam momentum spread), about $76 \%$ beam will benefit from the tune jumps above $G \gamma=19$. For tune jumps below $G \gamma=12$, more than $76 \%$ beam will benefit, as shown in Fig. 10. Moreover, the benefit portion of beam around transition is smaller, due to the larger momentum spread.

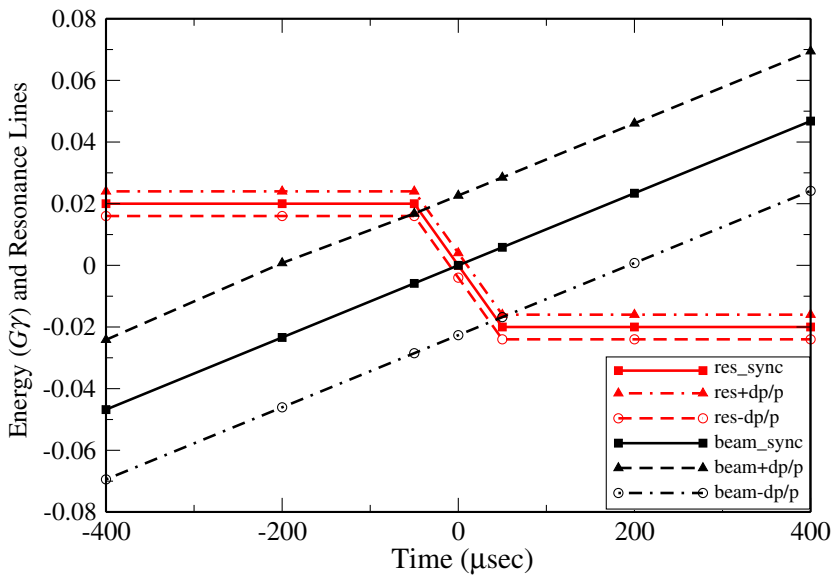

FIG. 8. Sketch of a resonance crossing on the increase $\nu_{x}$ side of the pulse $\left(G \gamma=54-\nu_{x}=45.3\right)$. The horizontal axis is relative time to the resonance crossing. The vertical axis is energy relative to the synchronous particle at jump time in unit of $G \gamma$. The solid line applies to the synchronous particle, and the two types of broken lines apply to the boundary particles at the \pm FWHM. The resonance lines (red) are given by Eq. (4) for positive and negative momentum spreads. The momentum FWHM width is assumed as $10^{-3}$, which is close to the real $d p / p$ at late part of the ramp. The chromaticity of -8 is used for the plot. The ramp rate $d G \gamma / d t$ is $0.117 / \mathrm{ms}$. The plot shows that particles within FWHM (76\% beam) benefit from the tune jump in this case.

Leaving horizontal tune jump quads off around transition has limited disadvantage.

One cannot change chromaticity fast enough (in a few $\mathrm{ms}$ ) to make the tune jump always in the slightly beneficial percentage. With the same sign of chromaticity, it is always

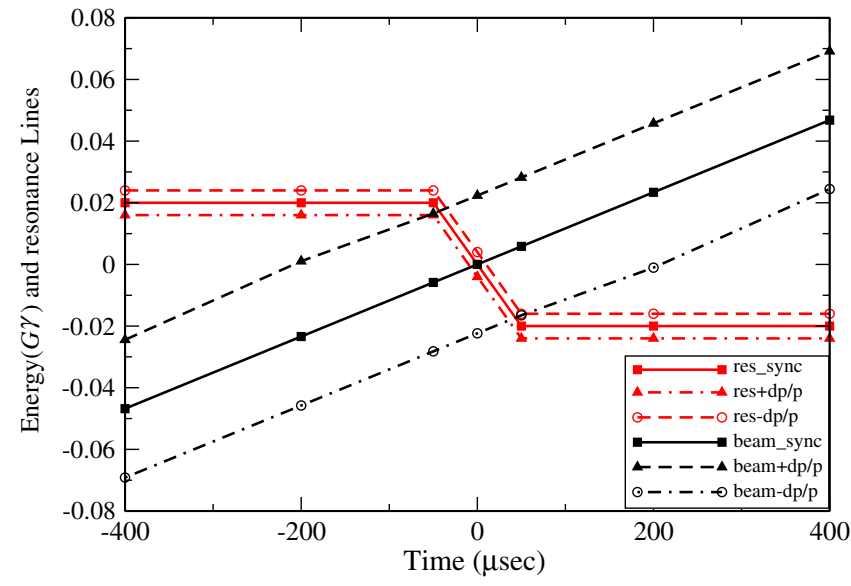

FIG. 9. This plot is similar to Fig. 8 except it is for $G \gamma=$ $36+\nu_{x}=44.7$ resonance. The resonance lines (red) are given by Eq. (5) for positive and negative momentum spreads. Note that in this case, the locations of positive and negative momentum spreads boundaries are reversed in comparison to the case of $G \gamma=54-\nu_{x}=45.3$. The portion of beam that benefits from the tune jump is only slightly smaller. Still, the plot shows that particles within FWHM (76\% beam) benefit from the tune jump in this case. 


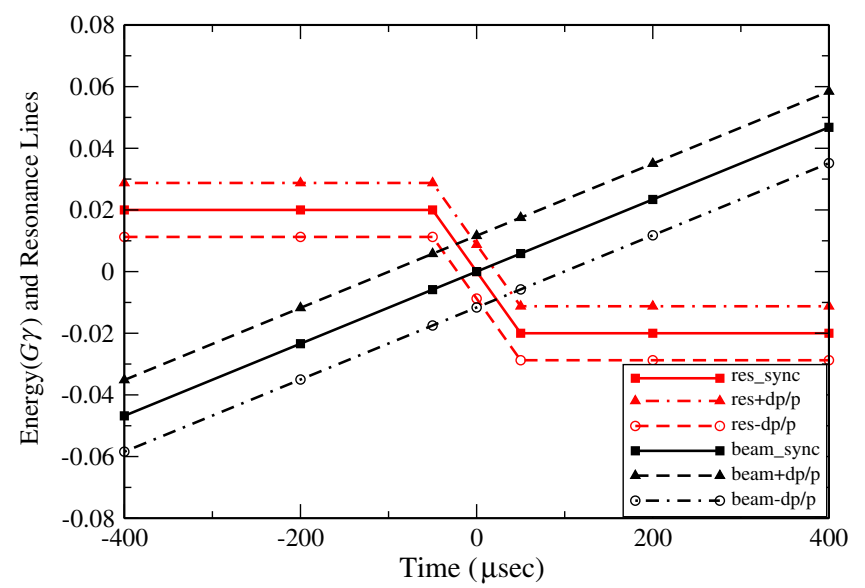

FIG. 10. Sketch of the resonance crossing on the increase $\nu_{x}$ side of the pulse at $\operatorname{low} \operatorname{energy}\left(G \gamma=18-\nu_{x}=9.3\right)$. The momentum FWHM width is assumed as $2.5 \times 10^{-3}$, which is close to the real $d p / p$ here. In the lower energy case, the beam $G \gamma$ spread is less important. Beam particles outside the FWHM benefit from the tune jump. The chromaticity of -7 is used for the plot.

true that more particles will benefit from the tune jump in one case (such as $\mathrm{N}-\nu_{x}$ ), but less from the other one (such as $N+\nu_{x}$ ). With negative chromaticity, one can see that more particles are benefiting from the tune jumps for $N-\nu_{x}$ resonances than the ones for $N+\nu_{x}$. However, the resonance width is dominated by the spread in $G \gamma$ (Figs. 8-9), benefit of fast chromaticity change is too small to be considered.

The jump timing determination requires accurate determination of beam energy as function of ramp time. The beam energy information on the energy ramp comes from measuring the AGS main magnetic field and measuring the beam momentum offset using the radial average from the beam position measuring system. As a cross check, the second set of beam energy information is derived from beam frequency and beam path length. The jump timing is then derived from the beam energy and horizontal tune as a function of ramp time.

From these sketch plots, one can estimate how accurate the jump quad timing has to be for the given momentum spread and ramp rate. Although the tune jump duration is $100 \mu \mathrm{s}$, some portion of the beam can still benefit from the tune jump with an offset of jump timing larger than $100 \mu$ s. For FWHM width of 0.04 (typical value after $G \gamma=19$ ) and the ramp rate of $0.117 / \mathrm{ms}$, the FWHM time width of the beam is $0.04 /(0.117 / \mathrm{ms})=0.341 \mathrm{~ms}$. Assuming Gaussian longitudinal distribution, the $\sigma$ of such a distribution should be $145 \mu \mathrm{s}$. For the case below transition, where FWHM of $d p / p$ is less than 0.04 , the $\sigma$ should be even smaller. Moreover, the horizontal intrinsic resonances can be treated as isolated resonances due to their spacing and weak strength. The final polarization is a product of Froissart-Stora formula for each resonance crossing.

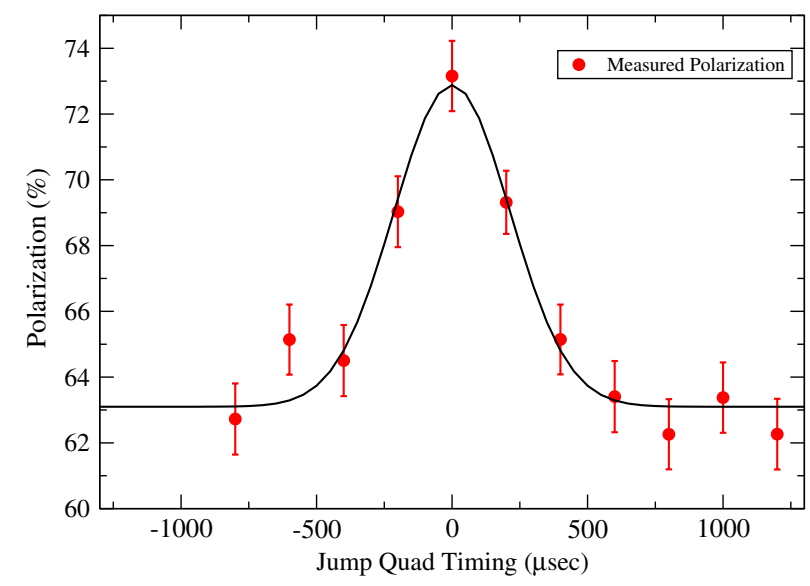

FIG. 11. The measured polarization at AGS extraction as function of overall jump quad timing. Error bars are statistical errors only. The solid line is a fit of Gaussian distribution with $\sigma=214 \mu \mathrm{s}$.

Assuming a Gaussian distribution in the longitudinal dimension, the polarization as function of overall jump timing shift should be close to a Gaussian distribution. The $\sigma$ of the Gaussian distribution should be around $145 \mu \mathrm{s}$. If there are errors in the individual jump quad timing, the distribution $\sigma$ will be larger.

Final polarization at AGS flattop was actually measured as function of overall jump timing for $2 \times 10^{11}$ bunch intensity. The measured timing scan curve is shown in Fig. 11 and it clearly demonstrates the benefit on polarization from the tune jump. When the tune jump timing is off by more than $500 \mu$ s or no particle benefits from the tune jump, the polarization level is at $63 \%$. When the tune jump timing is at its ideal value, polarization is about $73 \%$. A Gaussian fit shows $\sigma=214 \mu$ s, which is slightly larger than the estimation based on the known beam parameters. This implies that there are some tune jump timing errors. Further improvement on tune jump timing is possible. Fig. 11 also shows that the overall jump timing accuracy has to be within $\pm 100 \mu$ s level for the full benefit of the tune jump.

\section{CONCLUSIONS}

Vertical intrinsic resonances and imperfection resonances have been avoided by introducing two partial snakes in the AGS. But the partial snake magnets also move the stable spin direction away from the vertical and consequently excite depolarizing resonances associated with horizontal tune, so-called horizontal intrinsic resonances. They are generally weak but could cause accumulated polarization loss if left uncorrected. A modest horizontal tune jump system has been used to overcome these weak but numerous resonances while maintaining the transverse emittances. A relative gain of $15 \%$ polarization has been achieved with the tune jump system. The keys for the 
system are the accurate control of the jump quad timing and the elimination of any emittance growth. It can also be seen from the results that a slightly larger tune jump (such as 0.06) can benefit more particles. This scheme paves the way to use partial snakes to preserve polarization in the medium energy synchrotrons without the concern of losing polarization due to additional horizontal intrinsic resonances.

\section{ACKNOWLEDGMENTS}

The authors would like to thank all coordinators and operators in the main control room for their help in the machine tuning, all engineers and technicians who have contributed to the polarized proton development. This work was funded by the DOE of United States with Contract No. DE-AC02-98CH1-886.

[1] T. Khoe et al., Part. Accel. 6, 213 (1975); J. L. Laclare et al., J. Phys. (Paris), Colloq. 46, C2-499 (1985); H. Sato, D. Arakawa, S. Hiramatsu, Y. Mori, K. Ikegami, A. Takagi, T. Toyama, A. Ueno, and K. Imai, Nucl. Instrum. Methods Phys. Res., Sect. A 272, 617 (1988).

[2] F. Z. Khiari et al., Phys. Rev. D 39, 45 (1989).

[3] A. Lehrach et al., AIP Conf. Proc. 667, 30(2003).

[4] Ya.S. Derbenev and A. M. Kondratenko, Part. Accel. 8, 115 (1978).

[5] A. D. Krisch et al., Phys. Rev. Lett. 63, 1137(1989).
[6] I. Alekseev et al., Nucl. Instrum. Methods Phys. Res., Sect. A 499, 392 (2003).

[7] T. Roser, in Proc. of the 8th International Symposium on High-Energy Spin Physics, Minneapolis, 1988, AIP Conf. Proc. No. 187 (AIP, New York, 1989), p. 1442.

[8] H. Huang et al., Phys. Rev. Lett. 73, 2982 (1994).

[9] H. Huang et al., Phys. Rev. Lett. 99, 154801 (2007).

[10] F. Lin et al., Phys. Rev. ST Accel. Beams 10, 044001 (2007).

[11] N. Tsoupas, H. Huang, W. MacKay, F. Meot, T. Roser, and D. Trbojevic, Phys. Rev. ST Accel. Beams 16, 043501 (2013).

[12] M. Froissart and R. Stora, Nucl. Instrum. Methods 7, 297 (1960).

[13] V. Schoefer, L. A. Ahrens, K. A. Brown, J. W. Glenn, H. Huang, in Proceedings of the 24th Particle Accelerator Conference, PAC-2011, New York, 2011 (IEEE, New York, 2011), p. 1534.

[14] G. Atoian, A. Zelenski, A. Boghdanov, D. Raparia, M. Runtso, D. Steski, and V. Zajic, Proceedings of XVth International Workshop of Polarized Sources, Targets, and Polarimetry (PSTP), Charlottesville, 2013, http://pos.sissa .it/archive/conferences/182/052/PSTP2013_052.pdf.

[15] A. Poblaguev et al., Proceedings of XVth International Workshop of Polarized Sources, Targets, and Polarimetry (PSTP), Charlottesville, 2013, http://pos.sissa.it/archive/ conferences/182/053/PSTP2013_053.pdf.

[16] J. W. Glenn et al., in Proceedings of the 23rd Particle Accelerator Conference, Vancouver, Canada, 2009 (IEEE, Piscataway, NJ, 2009), p. 1699.

[17] S. Y. Lee and S. Tepikian, Phys. Rev. Lett. 56, 1635 (1986). 